\title{
A FAST EVALUATION OF INITIAL CONFIGURATIONS IN REPEATABLE INVERSE KINEMATICS FOR REDUNDANT MANIPULATORS
}

\author{
IGNACY DULEBA $^{a, *}$, IwONA KARCZ-DULEBA ${ }^{a}$, ARKADIUSZ MIELCZAREK $^{a}$ \\ ${ }^{a}$ Faculty of Electronics \\ Wrocław University of Science and Technology, Janiszewskiego 11/17, 50-372 Wrocław, Poland \\ e-mail: \{ignacy.duleba, iwona.duleba, arkadiusz.mielczarek\}@pwr.edu.pl
}

\begin{abstract}
A repeatable inverse kinematic task in robot manipulators consists in finding a loop (cyclic trajectory) in a configuration space, which corresponds to a given loop in a task space. In the robotic literature, an entry configuration to the trajectory is fixed and given by a user. In this paper the assumption is released and a new, indirect method is introduced to find entry configurations generating short trajectories. The method avoids a computationally expensive evaluation of (infinite) many entry configurations for redundant manipulators (for each of them, repeatable inverse kinematics should be run). Some fast-to-compute functions are proposed to evaluate entry configurations and their correlations with resulting lengths of trajectories are computed. It appears that only an original function, based on characteristics of a manipulability subellipsoid, properly distinguishes entry configurations that generate short trajectories. This function can be used either to choose one from a few possible entry configurations or as an optimized function to compute the best initial configuration.
\end{abstract}

Keywords: manipulator, repeatable inverse kinematics, initial configuration, optimization.

\section{Introduction}

For a few decades robots have been working in industry to perform dull, dangerous and precision tasks. As a rule, tasks for an industrial robot are described in its task space (X-space) while controls are planned and executed in a configuration space (Q-space). Most of the tasks are cyclic in the $\mathrm{X}$-space as a loop to follow (e.g., while welding, painting) is determined by technological requirements. In order to reduce production costs, there is a tendency to reduce also volumes of robotic cells and to make the surrounding of a robot crowdy. Therefore, cyclic trajectories are sought so that they correspond to cycles, X-loops, in an X-space. A collision-free motion in one cycle guarantees also collision avoidance in all cycles to come.

In the robotic literature this task, called repeatable inverse kinematics (Chiaverini et al., 2008), has a long history (Roberts and Maciejewski, 1993). Two main approaches to solve the task can be distinguished when an initial configuration, corresponding to the initial point of the X-loop, is also known. The first advises to expand original kinematics to non-redundant,

* Corresponding author augmented kinematics and to take advantage of the uniqueness of the inverse augmented kinematics as long as a planned trajectory does not meet any singular configuration (Roberts and Maciejewski, 1993; Klein et al., 1995). Augmenting the original kinematics can be performed in many ways, especially when a redundancy (the difference between the dimensions of the $\mathrm{Q}$ and $\mathrm{X}$-spaces) is significant. Functions extending original kinematics can be selected to optimize some performance criteria (Tchoń et al., 2009; Karpińska, 2012), e.g., to resemble trajectories generated with pseudo-inverse Jacobian matrix in the Newton algorithm of inverse kinematics (Nakamura, 1991). It is known that, locally, the trajectories minimize displacements in Q-space preserving desirable effect in X-space. Without any simplifications, this approach is difficult to apply as it requires searching for unknown partial differentials (derivatives of unknown functions with respect to configuration variables) (Tchon et al., 2009) that optimize a performance index over a given region in a Q-space. It is more tractable when unknown functions are expressed in a parametric form as linear combinations of parameters with some basis functions (polynomials, harmonics). In this case, an optimization is performed in a multi-dimensional 
space of parameters (Karpińska, 2012). Recently this approach has been improved to make the region task dependent (Duleba and Karcz-Duleba, 2017).

The other approach to solve repeatable inverse kinematics with a known initial configuration exploits continuation methods (Richter and DeCarlo, 1983). The paradigm has been successfully applied in robotics mainly to plan paths of mobile robots (Quinlan and Khatib, 1993). Repeatable inverse kinematics were solved using continuation methods by Duleba and Opałka (2013) with an elastic band algorithm. In a nutshell, implementation details of the method (Algorithm 1) will be recalled later on as the algorithm generates key data, Q-path lengths, corresponding to given initial configurations. Below, only the main idea of the method will be outlined. In an iterative process, the method constructs a sequence of loops in Q-space (Q-loops). Although reshaped, Q-loops are invariant in the method (the concept of invariance is frequently used in other tasks of robotics, too (Németh et al., 2016). Images of Q-loops (via forward kinematics) are closer and closer to a given cyclic path in X-space (X-loop). In this method a Newton algorithm is exploited with an optimization in a null space of the Jacobian matrix (Nakamura, 1991).

The aforementioned two approaches were compared by Duleba and Karcz-Duleba (2018). It appears that the second one over-performs the first as it runs faster, generates shorter trajectories, does not introduce extra singular configurations and is easy to implement. A fixed initial configuration assumption in repeatable inverse kinematics was relaxed by Duleba and Karcz-Duleba (2016). It appears that the configuration has got a huge impact on the quality (length) of the resulting Q-loop. However, selecting an optimal initial configuration is a hard numeric problem as it involves a multi-dimensional optimization and an evaluation of each trial point within the space requires to solve a time-consuming repeatable inverse kinematic task with a fixed entry configuration.

The main goal of this paper is to check whether it is possible to simplify this task and to determine factors that depend on initial configurations and correlate with the lengths of the resulting Q-loops. The answer to this question is important while a few possible initial configurations should be evaluated on-line and there is no time to solve a repeatable inverse kinematic task for each of them. The other possible application is to incorporate the factors into the steepest descent algorithm while searching for the best configuration within a space of all possible initial configurations.

The paper is organized as follows. In Section 2 a repeatable inverse kinematic task is stated formally and an elastic band algorithm, solving its version with the initial configuration fixed, is recalled. The algorithm serves as a tool for getting a reasonable amount of pairs: an initial configuration - the length of a Q-loop corresponding to the configuration. In Section 3 a few functions, derived from a Jacobian matrix (manipulability matrix) of a robot, are introduced to evaluate initial configurations. The functions describe an efficiency of a local motion in X-space around an initial configuration in averaged, optimistic and pessimistic sense and do not take into account the shape of a given X-loop. To retrieve this dependence, an original function is proposed to evaluate initial configurations according to privileged directions of motion within X-space. To construct this function, an algorithm of a manipulability ellipsoid intersection with a hyper-plane was developed. In Section 4 standard statistical tools are provided to verify correlations of proposed functions, evaluated at initial configurations, with the lengths of the resulting Q-loops. In Section 5 simulation results are presented. The data for simulations were collected from planar and spatial pendula manipulators tracing selected X-loops. Section 6 concludes the paper.

\section{Repeatable inverse kinematics}

Forward kinematics

$$
\boldsymbol{q} \rightarrow \boldsymbol{k}(\boldsymbol{q})=\boldsymbol{x}, \quad \operatorname{dim}(\boldsymbol{q})=n \geq m=\operatorname{dim}(\boldsymbol{x}),
$$

transform a configuration $\boldsymbol{q}$ from a configuration space $\mathbb{Q}$ to a point $\boldsymbol{x}$ in a task space $\mathbb{X}$ (Spong and Vidyasagar, 1989) which is a subset of the special Euclidean group $\mathbb{S E}(3)$. In this paper only redundant manipulators are considered and the redundancy index equals $r=n-m>$ 0 . In the task space, a closed path (X-loop) is given,

$$
\left\{\boldsymbol{x}(s), s \in\left[0, s_{\max }\right], \boldsymbol{x}(0)=\boldsymbol{x}\left(s_{\max }\right)\right\},
$$

where $s$ denotes the current path length. In the configuration space an initial configuration $\boldsymbol{q}(0)$ is defined which satisfies $\boldsymbol{k}(\boldsymbol{q}(0))=\boldsymbol{x}(0)$. A repeatable inverse kinematics task is to determine a cyclic path $\boldsymbol{q}(\cdot) \in \mathbb{Q}$ (Q-loop) which corresponds to X-loop (2), i.e.,

$$
\forall_{s \in\left[0, s_{\max }\right]} \boldsymbol{k}(\boldsymbol{q}(s))=\boldsymbol{x}(s), \quad \boldsymbol{q}(0)=\boldsymbol{q}\left(s_{\max }\right) .
$$

It appears that the most effective method of solving the task (Duleba and Karcz-Duleba, 2018) is based on the continuation principle (Richter and DeCarlo, 1983). Main steps implementing this method are presented in Algorithms 1 and 2 .

\section{Evaluations}

3.1. At a given configuration. To evaluate a given configuration, some measures should be defined. Most of them are based on a Jacobian matrix. At a particular configuration, the Jacobian $\boldsymbol{J}$ becomes a matrix of real numbers and it can be decomposed using the singular 
$\overline{\text { Algorithm 1. Implementation of the elastic band method }}$ in repeatable inverse kinematics.

Step 1. Read in a prescribed X-loop (2) and an initial (current) sequence of (s-value, configuration) pairs $L_{\text {disc }}=\left((0, \boldsymbol{q}(0)),\left(s_{\max }, \boldsymbol{q}(0)\right)\right)$. The initial Q-loop is trivial as it is composed of just a single point.

Step 2. Interpolate linearly points in $L_{\text {disc }}$ to get a continuous Q-loop, $L_{\text {cont }}(\cdot)$.

Step 3. Compute the distance between the image (via forward kinematics) of $L_{\text {cont }}(\cdot)$ and the given X-loop (2)

$$
\operatorname{err}\left(L_{\text {cont }}(\cdot)\right)=\int_{s=0}^{s_{\max }}\left\|\boldsymbol{x}(s)-\boldsymbol{k}\left(L_{\text {cont }}(s)\right)\right\| \mathrm{d} s,
$$

where $\|\cdot\|$ is a norm in $\mathbb{X} \subset \mathbb{S E}(3)$.

Step 4. If the error is below an acceptable threshold $\rho$, stop the computations and output $L_{\text {cont }}(\cdot)$. Otherwise progress with Step 5.

Step 5. Modify $L_{\text {disc }}$ the discrete version of Q-loop.

Step 6. Continue with Step 2.

value decomposition, or SVD (Golub and Reinsch, 1970), into

$$
\boldsymbol{J}=\boldsymbol{U}\left[\begin{array}{ll}
\boldsymbol{D} & \mathbf{0}
\end{array}\right] \boldsymbol{V}^{T},
$$

where rotation matrices $\boldsymbol{U} \in S O(m)(S O(m)$ denotes an orthogonal group of order $m), \boldsymbol{V} \in S O(n), \boldsymbol{D}=$ $\operatorname{diag}\left(d_{i}\right), i=1, \ldots, m$ is the diagonal $m \times m$ matrix collecting (non-negative) singular values $d_{i}$ and $\mathbf{0}$ is a zero matrix. Equation (7) admits a nice physical interpretation. At first, a sphere $\|\dot{\boldsymbol{q}}\|=1$ (or more precisely, a constant small radius sphere) in a tangent space to the configuration space (to which velocities $\dot{\boldsymbol{q}}$ belong to) is rotated by the matrix $V^{T}$ in $\mathbb{R}^{n}$. Then the sphere is scaled and projected onto $\mathbb{R}^{m}$ to get a manipulability ellipsoid with semi-axes lengths equal to $d_{i}$. Finally, the ellipsoid is rotated with the matrix $\boldsymbol{U}$ in $\mathbb{R}^{m}$ to get another ellipsoid.

This construction shows how to map a fixed displacement from $\mathbb{Q}$ space into the corresponding one in $\mathbb{X}$ space. Notice that displacements are equivalent to velocities when a motion time is fixed and short. From a practical point of view, it is more important to get the inverse relationship, i.e., the displacement in $\mathbb{Q}$-space corresponding to a fixed motion in $\mathbb{X}$-space,

$$
\boldsymbol{J}^{\#}=\boldsymbol{V}\left[\begin{array}{c}
\boldsymbol{D}^{-1} \\
\mathbf{0}^{T}
\end{array}\right] \boldsymbol{U}^{T},
$$

where $\boldsymbol{D}^{-1}=\operatorname{diag}\left(d_{i}^{-1}\right)$ is the inverse of $\boldsymbol{D}$.

A standard method to evaluate a given configuration is based on an $m \times m$ non-negative definite, symmetric manipulability matrix (Maciejewski and Klein, 1989)

$$
\boldsymbol{M}(\boldsymbol{q})=\boldsymbol{J}(\boldsymbol{q}) \boldsymbol{J}^{T}(\boldsymbol{q})=\boldsymbol{U} \boldsymbol{D} \boldsymbol{D}^{T} \boldsymbol{U}^{T}
$$

Algorithm 2. Details of Step 5.

Step 5a. On the path (2) determine the furthest point $\boldsymbol{x}\left(s^{\star}\right)$ from $\boldsymbol{k}\left(L_{\text {cont }}(\cdot)\right)$

$$
\left\|x\left(s^{\star}\right)-\boldsymbol{k}\left(L_{\text {cont }}\left(s^{\star}\right)\right)\right\|=\max _{s=0}^{s_{\max }}\left\|x(s)-\boldsymbol{k}\left(L_{\text {cont }}(s)\right)\right\| .
$$

Step 5b. Based on $s^{\star}$, within $L_{\text {disc }}$ select two neighbor pairs $\left(s_{j}, \boldsymbol{q}\left(s_{j}\right)\right),\left(s_{j+1}, \boldsymbol{q}\left(s_{j+1}\right)\right)$ such that $s_{j}<s^{\star}<$ $s_{j+1}$ and denote by $\boldsymbol{q}^{1}=\boldsymbol{q}\left(s_{j}\right), \boldsymbol{q}^{2}=\boldsymbol{q}\left(s_{j+1}\right)$ their configuration components.

Step 5c. Run the Newton algorithm with the optimization in the null space of the Jacobian matrix (Nakamura, 1991)

$$
\begin{aligned}
\boldsymbol{q}_{i+1}= & \boldsymbol{q}_{i}+\xi_{i} \cdot \boldsymbol{J}^{\#}\left(\boldsymbol{q}_{i}\right)\left(\boldsymbol{x}\left(s^{\star}\right)-\boldsymbol{k}\left(\boldsymbol{q}_{i}\right)\right) \\
& +\left.\eta_{i}\left(\boldsymbol{I}_{n}-\boldsymbol{J}^{\#}\left(\boldsymbol{q}_{i}\right) \boldsymbol{J}\left(\boldsymbol{q}_{i}\right)\right) \frac{\partial f}{\partial \boldsymbol{q}}\right|_{\boldsymbol{q}=\boldsymbol{q}_{i}},
\end{aligned}
$$

where

- $i$ is the iteration counter and superscript $T$ signifies matrix transposition,

- $\boldsymbol{J}=\mathrm{d} \boldsymbol{k} / \mathrm{d} \boldsymbol{q}$ denotes the Jacobian matrix,

- $\boldsymbol{J}^{\#}=\boldsymbol{J}^{T}\left(\boldsymbol{J} \boldsymbol{J}^{T}\right)^{-1}$ is the pseudo-inverse of $\boldsymbol{J}$,

- $\boldsymbol{x}\left(s^{\star}\right)$ is the current goal point,

- $\xi_{i}, \eta_{i}$ are positive parameters,

- an initial configuration

$$
\boldsymbol{q}_{0}=\lambda \cdot \boldsymbol{q}^{1}+(1-\lambda) \cdot \boldsymbol{q}^{2}
$$

where

$$
\lambda=\frac{\left(s^{\star}-s_{j}\right)}{\left(s_{j+1}-s_{j}\right)},
$$

- the optimized function $f(\boldsymbol{q})=\left\|\boldsymbol{q}-\boldsymbol{q}^{1}\right\|+\left\|\boldsymbol{q}-\boldsymbol{q}^{2}\right\|$.

The Newton algorithm is completed when an error $\left\|\boldsymbol{k}\left(\boldsymbol{q}_{i}\right)-\boldsymbol{x}\left(s^{\star}\right)\right\|$ drops below a given threshold $\delta$.

Step 5d. Result of the Newton algorithm run: a pair $\left(s^{\star}, q\left(s^{\star}\right)\right)$, satisfying $k\left(q\left(s^{\star}\right)\right)=x\left(s^{\star}\right)$, is added into the appropriate place in $L_{\text {disc }}$ to preserve the ordering of the sequence with respect to the variable $s$.

From Eqn. (9), the manipulability index

$$
\boldsymbol{\rho}(\boldsymbol{q})=\sqrt{\operatorname{det}(\boldsymbol{M}(\boldsymbol{q}))}=\prod_{i=1}^{m} d_{i}(\boldsymbol{q})
$$

is derived, which is proportional to the volume of the manipulability ellipsoid. The manipulability index evaluates an averaged behavior of the manipulator when all its possible local directions of motion are uniformly distributed within $\mathbb{R}^{m}$. The index (10) allows one to determine the most and the worst promising displacements in $\mathbb{X}$-space when $\dot{\boldsymbol{q}}$ is restricted and small 
(only in this case a Jacobian matrix reasonably describes the infinitesimal behavior of the manipulator). The displacements correspond to $d_{\max }, d_{\min }$, respectively,

$$
d_{\min }(\boldsymbol{q})=\min _{i=1}^{m} d_{i}(\boldsymbol{q}), \quad d_{\max }(\boldsymbol{q})=\max _{i=1}^{m} d_{i}(\boldsymbol{q}) .
$$

The minimal singular value $d_{\min } \geq 0$ and the manipulability index (10) sometimes serve as detectors of singular configurations. At those configuration both indices attain the value of zero.

Indices (10) and 111) are averaged, pessimistic and optimistic predictions of motion quality in $\mathbb{X}$-space. Their reciprocals $1 / d_{\min }, 1 / d_{\max }$, and $1 / \rho$ are more appropriate to evaluate motion in $\mathbb{Q}$-space.

The indices (10) and (11) or their inverses do not cover a real motion in $\mathrm{X}$-space along a particular direction or a sub-space of possible directions. The directions are available when X-loop (3) is given. To cover also this case, a manipulability ellipsoid will be sectioned at a selected configuration with a hyper-plane passing through the origin of $\mathbb{R}^{m}$ and spanned by some vectors forming a basis for a sub-space of predicted directions.

3.2. Manipulability sub-ellipsoid. Assume that a set of $j \leq m m$-dimensional orthonormal versors (unit-length vectors perpendicular to each other) $\boldsymbol{b}_{i}, i=$ $1, \ldots, j$ anchored at the origin of $\mathbb{R}^{m}$ are given. The vectors can be arranged into an $m \times j$ matrix $\boldsymbol{B}$, which satisfies $\operatorname{rank}(\boldsymbol{B})=j$, and span a $j$-dimensional hyper-plane.

At a given configuration $\boldsymbol{q}$, a Jacobian matrix $\boldsymbol{J}(\boldsymbol{q})$ is computed and expanded with the SVD algorithm as in Eqn. (7). As we are interested in intersection of an $m$-dimensional ellipsoid with a $j$-dimensional hyper-plane, only the term $\boldsymbol{U} \boldsymbol{D}$ is important. Vectors $\boldsymbol{B}$ rotated with matrix $\boldsymbol{U}^{T}$ and scaled with $\boldsymbol{D}^{T}=\boldsymbol{D}$,

$$
\boldsymbol{B}^{T} \boldsymbol{U} \boldsymbol{D}=\left(\boldsymbol{D}^{T} \boldsymbol{U}^{T} \boldsymbol{B}\right)^{T}=\boldsymbol{J}_{B},
$$

define a $j \times m$ matrix $\boldsymbol{J}_{B}$. This matrix is expanded with the SVD algorithm into

$$
\boldsymbol{J}_{B}=\tilde{\boldsymbol{U}} \tilde{\boldsymbol{D}} \tilde{\boldsymbol{V}}^{T}
$$

to form a sub-ellipsoid in $\mathbb{R}^{j}$. For this sub-ellipsoid, a manipulability index, cf. Eqn. $(10), \tilde{\boldsymbol{\rho}}=\prod_{i=1}^{j} \tilde{d}_{i}$ evaluates a motion ability in a hyper-plane spanned by vectors $\boldsymbol{B}$.

Three remarks concerning construction of the sub-ellipsoid are important:

1. $\boldsymbol{b}_{i}, i=1, \ldots, j$ should be unit-length vectors. To prove this, let us assume that $\boldsymbol{U}$ in Eqn. (12) is the identity matrix $\boldsymbol{I}_{m}$ and there is only one vector $\boldsymbol{b}_{1}=$ $(\alpha, 0, \ldots, 0)^{T}$ with $\alpha \neq 0$ spanning a line. In this trivial case, one can expect that the manipulability sub-ellipsoid will have only one singular value equal $d_{1}$. However, from Eqn. (13) one gets $\tilde{\boldsymbol{U}}=\boldsymbol{I}_{1} \tilde{\boldsymbol{V}}=$ $\boldsymbol{I}_{m}$ and the only singular value equals $\alpha d_{1}$. Thus $\|\alpha\|=1$ and $\boldsymbol{b}_{1}$ is a unit-length vector.

2. The set $\boldsymbol{b}_{i}, i=1, \ldots, j$ should be orthonormal. Once again, assume that $\boldsymbol{U}=\boldsymbol{I}_{m}$ and $\boldsymbol{b}_{1}=(1,0, \ldots, 0)^{T}$, $\boldsymbol{b}_{2}=(0,1,0, \ldots, 0)^{T}$. Direct calculations show, as expected, that the diagonal elements of $\boldsymbol{D}$ and $\tilde{D}$ coincide for $i=1,2$. For another set of unit-length, non-perpendicular vectors $\boldsymbol{b}_{1}=(1,0, \ldots, 0)^{T}, \boldsymbol{b}_{2}=$ $(1 / \sqrt{2}, 1 / \sqrt{2}, 0, \ldots, 0)^{T}$ the required property does not hold.

3. The vectors $\boldsymbol{b}_{i}, i=1, \ldots, j$ can be rotated simultaneously around any hyper-plane perpendicular to the hyper-plane spanned by original $\boldsymbol{B}$ and passing through the origin of $\mathbb{R}^{m}$. In other words, the semi-axes of the constructed sub-ellipsoid are invariant with respect to these particular rotations of the orhonormal set $\boldsymbol{B}$. Any such rotation influences $\tilde{\boldsymbol{U}}$ and $\tilde{\boldsymbol{V}}^{T}$ in Eqn. (13) but not $\tilde{D}$.

A dual construction of using $\boldsymbol{J}^{\#}$ instead of $\boldsymbol{J}$ is also possible and basis vectors $\boldsymbol{B}$ are mapped into $\mathbb{Q}$-space via

$$
\boldsymbol{V}\left[\begin{array}{c}
\boldsymbol{D}^{-1} \\
\mathbf{0}^{T}
\end{array}\right] \boldsymbol{U}^{T} \boldsymbol{B} .
$$

It is worth noticing that selecting for $\boldsymbol{B}$ the identity matrix $\boldsymbol{I}_{m}$ in Eqn. (14) gives just $\boldsymbol{J}^{\#}$, cf. Eqn. (8).

A special case is particularly important where $\boldsymbol{B}$ is composed of only one unit-length vector-direction $\boldsymbol{b}_{1}$. In this case one-dimensional subspace is defined with a manipulability given by the expression

$$
\frac{1}{d} \triangleq\left\|\boldsymbol{D}^{-1} \boldsymbol{U}^{T} \boldsymbol{b}_{1}\right\|
$$

3.3. Other evaluation functions. The performance indices from the previous subsections were defined for a single configuration only. For relatively long $\mathbb{X}$-loops, it is expected that configurations along the path will change significantly. To take into account also this fact and do not increase a computational effort substantially, we take another configuration, say $\boldsymbol{q}_{m}$, along the path corresponding to the most distant point from $\boldsymbol{x}(0)$. For $\boldsymbol{q}_{m}$ the same functions are evaluated as in $\boldsymbol{q}_{0}$ and the results are averaged to get the final evaluation of $\boldsymbol{q}_{0}$. In order to compute $\boldsymbol{q}_{m}$, it is enough to run the very first step of the elastic band algorithm presented previously.

\section{Correlations}

Evaluation functions $e_{i}, i=1,2, \ldots$ give some characteristics of an initial configuration $\boldsymbol{q}_{0}$ possibly 
supplemented by another configuration $\boldsymbol{q}_{m}$ which also contributes to evaluation of $\boldsymbol{q}_{0}$. For each generated $\boldsymbol{q}_{0}$, the elastic band algorithm is run and the length of the $\mathbb{Q}$-loop corresponding to a given $\mathbb{X}$-loop is obtained. In this way a set of pairs $\left(L\left(\boldsymbol{q}_{0}\right), e_{i}\left(\boldsymbol{q}_{0}\right)\right)$ is available and the goal is to find whether the two variables, $L$ and $e_{i}$, are correlated or not.

Generally, a correlation is a number that defines how strong a relationship between two random variables ( $L$ and $e_{i}$ in our case) is. There are many types of correlation. However, this term is usually referred to the Pearson correlation coefficient, PCC (Dixon and Massey, 1969), defined as follows:

$$
\rho_{L, e}=\frac{\mathrm{E}\left[\left(L-\mu_{L}\right)\left(e-\mu_{e}\right)\right]}{\sigma_{L} \sigma_{e}},
$$

where ' $E$ ' denotes the expected value, $\mu_{Z}$ is the mean of $Z, \sigma_{Z}$ stands for the standard deviation of $Z$ and $e$ denotes one function from the set $e_{i}, i=1, \ldots$ For sample sets of random variables containing $N$ values, $\rho_{L, e}$ can be estimated with

$$
p_{L, e}=\frac{\sum_{i=1}^{N}\left(L_{i}-\bar{L}\right)\left(e_{i}-\bar{e}\right)}{\sqrt{\sum_{i=1}^{N}\left(L_{i}-\bar{L}\right)^{2}} \sqrt{\sum_{i=1}^{N}\left(e_{i}-\bar{e}\right)^{2}}},
$$

where $\bar{z}$ is the sample mean from the set $\left\{z_{1}, \ldots, z_{N}\right\}$. PCC is a number from the interval $[-1,1]$ quantifying a linear relationship between data sets (random variables). The values close to 0 means incoherence of variables, close to +1 (resp., -1 ) their strong, positive (resp., negative) linear dependence.

Due to the limitation of PCC in detecting non-linear relationships, in this paper also Spearman's rank correlation coefficient, SRCC (Dixon and Massey, 1969; Kendall and Gibbons, 1990) will be used. SRCC is simply PCC, where rough data-sets are overridden by values of their ranking functions,

$$
s_{L, e}=\frac{\mathrm{E}\left[\left(\operatorname{rg}(L)-\mu_{\operatorname{rg}(L)}\right)\left(\operatorname{rg}(e)-\mu_{\mathrm{rg}(e)}\right)\right]}{\sigma_{\operatorname{rg}(L)} \sigma_{\operatorname{rg}(e)}} .
$$

$\operatorname{rg}(\cdot)$ denotes a ranking function (each rough value is replaced with its index in an ordered sequence of all values of a given variable). SRCC is particularly well suited to detect monotonous relationship between variables. It attains the value of +1 (resp., -1 ) for increasing (resp., decreasing) functions.

\section{Simulations}

A first few simulations were carried out on models of $n$-dimensional planar pendula $\boldsymbol{q}=\left(q_{1}, \ldots, q_{n}\right)^{T}$ with a two-dimensional $(x, y)^{T}$ task-space, $\operatorname{dim} \mathbb{Q}>\operatorname{dim} \mathbb{X}$ described by kinematics

$$
(x, y)^{T}=\boldsymbol{k}(\boldsymbol{q})=\left(\sum_{i=1}^{n} a_{i} c_{1, i}, \sum_{i=1}^{n} a_{i} s_{1, i}\right)^{T} .
$$

In Eqn. (23), and later on, a standard robotic convention to denote sine/cosine functions was utilized, $c_{1, i}=$ $\cos \left(\sum_{j=1}^{i} q_{j}\right)$. All lengths of links are equal to $a_{i}=$ 1 [m]. The pendula are easy to visualize and a desired degree of redundancy can be obtained easily.

In Task 1, the 3-DoF pendulum traced forth and back a straight-line in $\mathbb{X}$-space between points $\boldsymbol{x}(0)=(2,0.1)^{T}$ and $\boldsymbol{x}(0.5)=\boldsymbol{x}(0)+(0, R)^{T}$ with parameter $R$ varied in the range from 0.1 to 1.5 . 150 initial configurations $\boldsymbol{q}_{0}$ were examined with evaluation functions derived from maximal and minimal singular values,

$$
e_{1}=\frac{1}{d_{1}}=\frac{1}{d_{\max }}, \quad e_{2}=\frac{1}{d_{2}}=\frac{1}{d_{\min }},
$$

from the manipulability index,

$$
e_{3}=\frac{1}{d_{1} d_{2}}
$$

and directional characteristics,

$$
e_{4}=\frac{1}{d}
$$

based on the vector $\boldsymbol{b}_{1}=(\boldsymbol{x}(0.5)-\boldsymbol{x}(0)) / \| \boldsymbol{x}(0.5)-$ $\boldsymbol{x}(0) \|=(0,1)^{T}$. Results depicted in Fig. 1 and others not presented here, lead to the following conclusions:

1. General purpose functions $e_{1}, e_{2}, e_{3}$, evaluated at $\boldsymbol{q}_{0}$, are not correlated with lengths of Q-loops (PCC, SRCC are relatively small and sometimes change their signs). The directional evaluation function $e_{4}$ is strongly and positively correlated with the Q-loop length. As predicted, correlation coefficients approach +1 for very short line $\mathrm{X}$-paths because, in this case, the Jacobian matrix at $\boldsymbol{q}_{0}$ is almost the same as for other configurations along the X-path.

2. By adding a trail configuration $\boldsymbol{q}_{m}$, a correlation between the values of the function $e_{4}$ and $\mathbb{Q}$-loop lengths is strengthened as weighted values at $\boldsymbol{q}_{0}$ and $\boldsymbol{q}_{m}$ better characterize the resulting $Q$-loop than a single initial $\boldsymbol{q}_{0}$. The improvement in the values of the correlation coefficients is negligible for other evaluation functions.

In Fig. 2 points on the plane: length of a $\mathbb{Q}$-loop $(L)$ vs. values of evaluated functions $\left(e_{i}\right)$, are visualized for a single value of parameter $R=0.2$ and all initial configurations $\boldsymbol{q}_{0}$ generated. There are no regularities in 


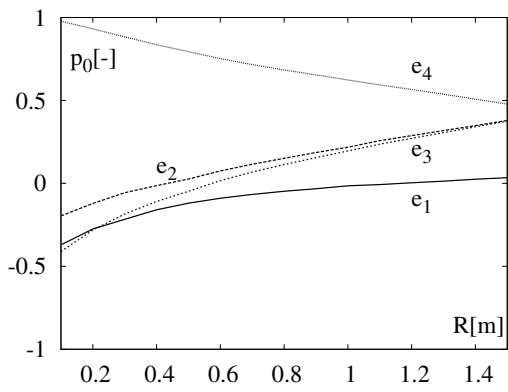

(a) PCC at $q_{0}$

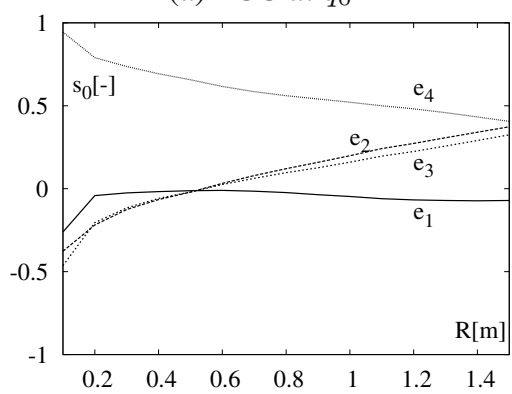

(b) SRCC at $q_{0}$

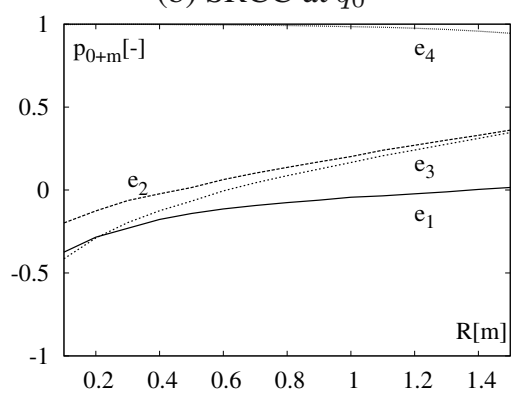

(c) PCC weighted at $q_{0}$ and $q_{m}$

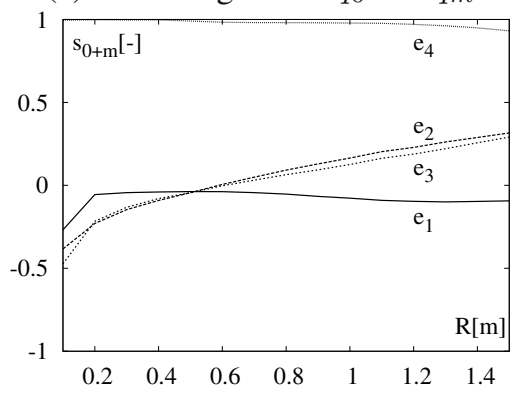

(d) SRCC weighted at $q_{0}$ and $q_{m}$

Fig. 1. Linear paths of the 3-DoF pendulum. Pearson (PCC) and Spearman (SRCC) correlation coefficients for pairs $\left(L, e_{i}\right)$ as a function of parameter $R$. Functions $e_{i}, i=$ $1, \ldots, 4$ evaluated either at $\boldsymbol{q}_{\mathbf{0}}\left((\mathrm{a})\right.$ and (b)) or at $\boldsymbol{q}_{\mathbf{0}}$ and $\boldsymbol{q}_{\boldsymbol{m}}((\mathrm{c})$ and (d)).

the set of pairs $\left(L, e_{i}\right)$ for $i=1,2,3$, Fig. 2- (a)-(c), but a linear dependence $\left(L, e_{4}\right)$ is clearly visible, cf. Fig. 2]d). For the same task, in Fig. 3 five postures $\boldsymbol{q}_{0}$ are presented generating the shortest and the longest $\mathbb{Q}$-loops. The

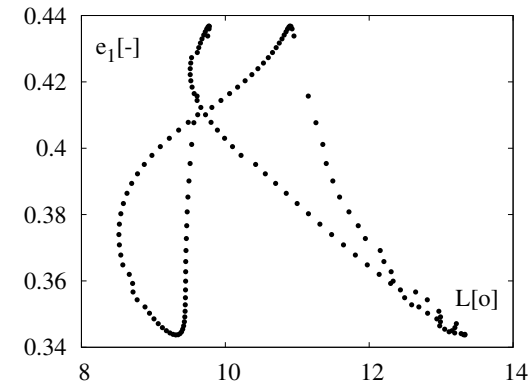

(a) $\left(L, e_{1}\right)$

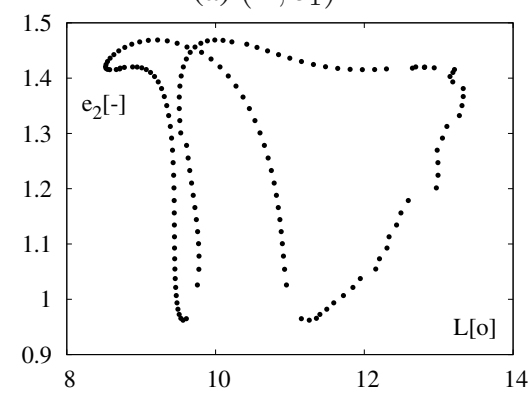

(b) $\left(L, e_{2}\right)$

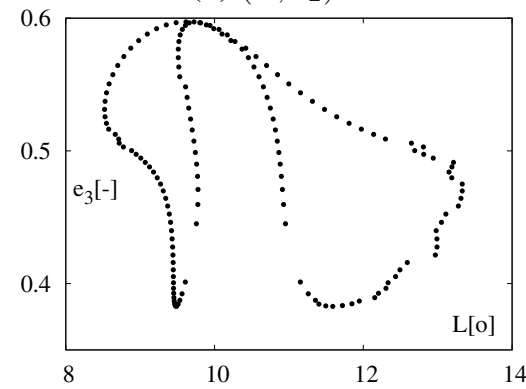

(c) $\left(L, e_{3}\right)$

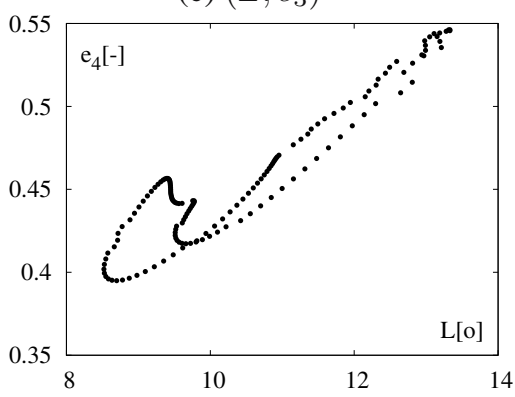

(d) $\left(L, e_{4}\right)$

Fig. 2. Linear $\mathbb{X}$-loop of the $3-\mathrm{DoF}$ pendulum. Points $\left(L, e_{i}\right)$, $i=1, \ldots, 4$, derived from many initial configurations $\boldsymbol{q}_{\mathbf{0}}$.

shortest loops were initialized at configurations requiring only motion of the first degree of freedom to perform a desired X-loop (the other DoFs making only minor corrections). The longest Q-paths were generated with the last two links arranged in almost a singular configuration $q_{3} \simeq 0^{\circ}$. In this case, to move the end-effector along 


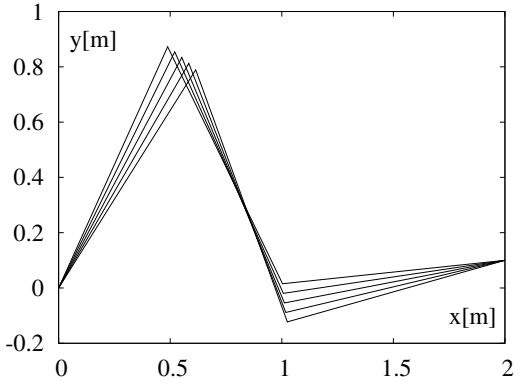

(a)

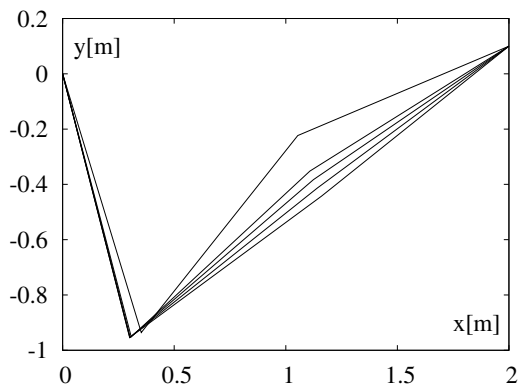

(b)

Fig. 3. Linear path of the 3-DoF pendulum. Initial postures $\boldsymbol{q}_{0}$ providing the shortest (a) and the longest (b) $\mathbb{Q}$-loops.

the $y$-axis, all components of $\boldsymbol{q}$ have to be changed significantly.

In Task 2 , rectangular $\mathbb{X}$-loops were selected to check the correlations with two directions privileged instead of one. A family of rectangles with a left bottom corner placed at $(2-R, 0.1)$ and shifts along axes $(\Delta x=$ $R, \Delta y=2 R$ ) was selected with parameter $R$ varied in the range from 0.1 to 0.8 with step 0.05 . In Fig. 4 only the $\left(L, e_{4}\right)$ correlation coefficients are presented (the other are less informative, being very similar to linear X-loops). In Fig. 4, and those to follow, characteristics marked as (0) were based on $\boldsymbol{q}_{0}$ while those marked as $(0+\mathrm{m})$ were based on $\boldsymbol{q}_{0}$ and $\boldsymbol{q}_{m}$. We have

$$
e_{4}=w_{1} \cdot e_{4}^{X}+w_{2} \cdot e_{4}^{Y},
$$

where $e_{4}^{X}$ was computed for the direction $\boldsymbol{b}_{1}=(1,0)^{T}$ while $e_{4}^{Y}$ for the direction $\boldsymbol{b}_{1}=(0,1)^{T}$. The weights were selected as the percentages of motion in those directions while tracing the rectangles, i.e., $w_{1}=1 / 3, w_{2}=2 / 3$. The observations made for the line path remain valid also for the rectangular X-paths.

In Task $3 \mathbb{X}$-line paths of the 5 -DoF pendulum were examined. A path joints point $\boldsymbol{x}(0)=(3.5,0.1)^{T}$ with $\boldsymbol{x}(0.5)=\boldsymbol{x}(0)+(0, R)^{T}$ for $R \in[0.1,2.5]$ discretized with step 0.1 . For each $R$, about 600 initial configurations $\boldsymbol{q}_{0}$ were generated. Results are presented in Fig. 5. Also in this case the regularities observed for the $3-\mathrm{DoF}$ pendulum

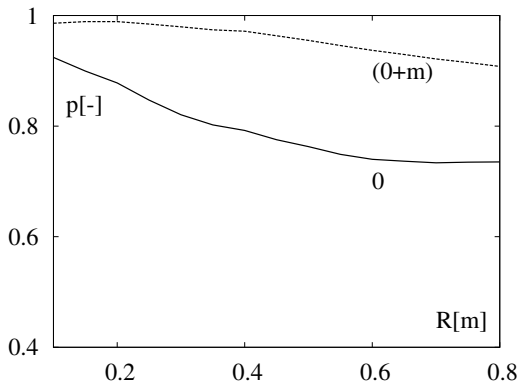

(a)

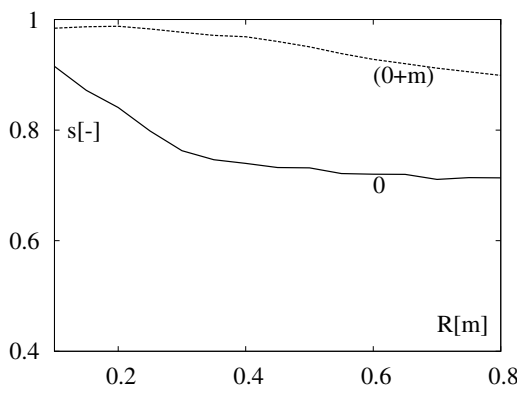

(b)

Fig. 4. Rectangular paths of the 3-DoF pendulum. Pearson (a) and Spearman (b) correlation coefficients for pair $\left(L, e_{4}\right)$ as a function of parameter $R$.

remain valid but the characteristics drop much faster than for the 3-DoF pendulum.

Table 1. 5-DoF pendulum, line path with $\boldsymbol{x}(0)=(R, 0.1)$ and $\boldsymbol{x}(0.5)=\boldsymbol{x}(0)+(0,2)^{T}$, for some values of $R$

\begin{tabular}{|c|c|c|c|c|c|}
\hline$R$ & $\boldsymbol{q}_{0}$ items & $\min$ & $\max$ & avg & $\sigma$ \\
\hline$[\mathrm{m}]$ & {$[-]$} & {$\left[^{\circ}\right]$} & $\left.{ }^{\circ}\right]$ & $\left.{ }^{\circ}\right]$ & {$\left[^{\circ}\right]$} \\
\hline \hline 2.5 & 2128 & 51.0 & 190.3 & 106.7 & 26.8 \\
3.0 & 1150 & 47.2 & 198.0 & 93.9 & 23.6 \\
3.5 & 614 & 44.7 & 141.2 & 86.8 & 20.0 \\
4.0 & 326 & 44.4 & 128.6 & 88.8 & 19.3 \\
\hline
\end{tabular}

One more aspect of varying initial configurations was checked for Task 3. Fixed length (equal to 2) line $\mathrm{X}$-loops were placed along the $y$-axis with the initial $\boldsymbol{x}(0)$ varied. For each $\boldsymbol{x}(0)$, a family of admissible initial configurations $\boldsymbol{q}_{0}$ were generated. Statistical data (minimal, maximal, average, and the standard deviation values of lengths of resulting $\mathbb{Q}$-loops) for this task are collected in Table1. It appears that the location of a task is important. The number of possible initial configurations is much bigger when the task is located relatively close to the base of the manipulator and decreases substantially when it is close to a boundary of the task space. Usually, a lot of initial configurations, being bent and twisted, are 


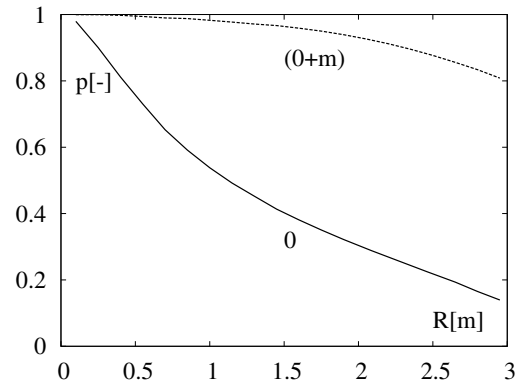

(a)

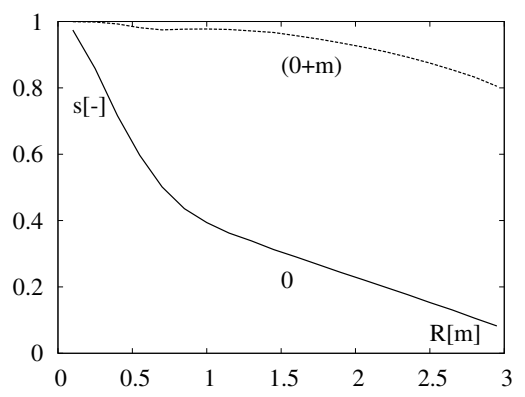

(b)

Fig. 5. Linear paths of the 5-DoF pendulum. Pearson (a) and Spearman (b) correlation coefficients for pair $\left(L, e_{4}\right)$ as a function of parameter $R$.

not particularly well suited to initialize short $Q$-loops. Moreover, in practical manipulators most of them are not admissible due to construction constraints. Based on a heuristic rule, one should locate a task far away from singular configurations to allow for flexibility in selecting possible $\mathbb{Q}$-loops either to optimize their lengths or to avoid possible obstacles. However, this just sketched task-location problem needs deeper theoretical studies.

The last two simulations were performed on a 4-DoF manipulator, composed of two 2-DoF planar pendula, with a three-dimensional task space. The second pendulum works on a plane perpendicular to the second link of the first pendulum. Kinematics of the manipulator are given by

$$
\left[\begin{array}{l}
x \\
y \\
z
\end{array}\right]=\boldsymbol{k}(\boldsymbol{q})=\left[\begin{array}{c}
a_{1} c_{1}+a_{2} c_{12}-r s_{12} \\
a_{1} s_{1}+a_{2} s_{12}+r c_{12} \\
a_{3} s_{3}+a_{4} s_{34}
\end{array}\right],
$$

where $r=a_{3} c_{3}+a_{4} c_{34}$.

Task 4 was to follow a line characterized by its initial point $\boldsymbol{x}(0)=(1,0,0.5)^{T}$, and the shift vector $\Delta_{1}=(R, R, R)^{T}$, where parameter $R \in(0.1,1.1)$ was varied with step 0.1 . In Fig. 6 correlation coefficients of resulting Q-loop lengths $L$ with directional function $e_{4}$ are presented as a function of $R$. For other functions $\left(e_{1}-e_{3}\right)$ the coefficients are relatively small and do not exceed the value of \pm 0.4 .

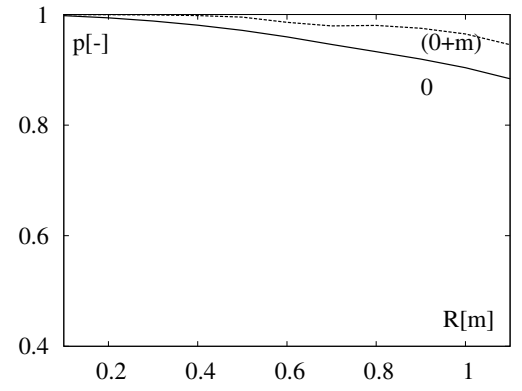

(a)

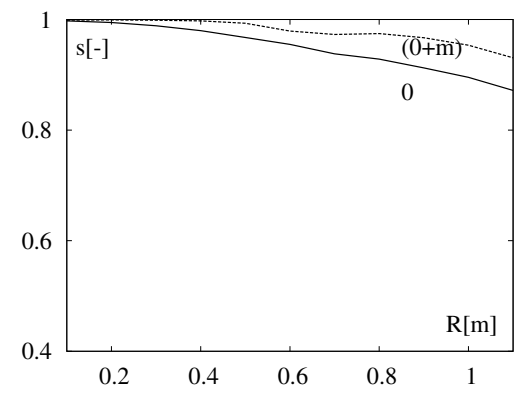

(b)

Fig. 6. Linear paths of the 2-DoF+2-DoF pendulum. Pearson (a) and Spearman (b) correlation coefficients for pair $\left(L, e_{4}\right)$ as a function of parameter $R$.

In Task 5 a parallelogram path was traced, anchored at $\boldsymbol{x}(0)$ and spanned by two vectors $\Delta_{1}$ and $\Delta_{2}=$ $(0, R, 0)^{T}$. The parameter $R \in(0.1,0.9)$ while the other data were selected as in Task 4 . The examined correlation coefficients are visualized in Fig.7.

All observations made for planar pendula (a two-dimensional task space) remain valid also for tree dimensional task spaces.

\section{Conclusions}

In this paper a task of a fast selection among initial configurations in repeatable inverse kinematics for redundant manipulators was solved. An initial configuration which provides the shortest possible loop in the configuration space is desirable but, due to computational time restrictions, the selection cannot be performed by solving the repeatable inverse kinematic task for each of possible initial configurations. Generally, for redundant manipulators, their number is infinite as the configurations belong to a multi-dimensional subspace of the configuration space. To avoid a computationally complex evaluation, an indirect method was proposed to search for the required initial configuration. Correlation between the length of the loop initialized at a given configuration and values of some (fast to compute) functions evaluated at the configuration was examined. 


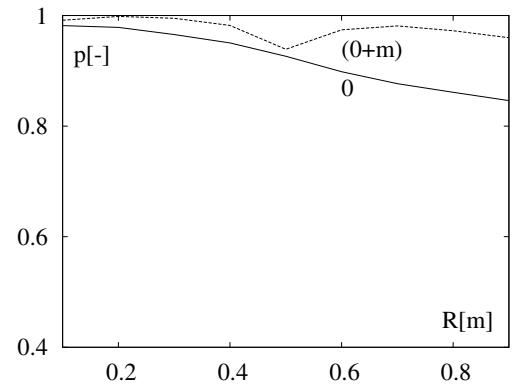

(a)

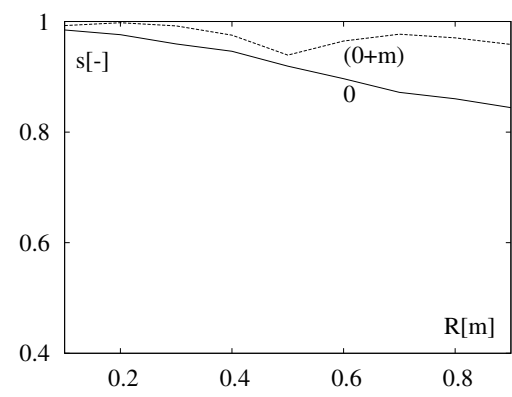

(b)

Fig. 7. Rectangular paths of the 2-DoF+2-DoF pendulum. Pearson (a) and Spearman (b) correlation coefficients for pair $\left(L, e_{4}\right)$ as a function of parameter $R$.

A local motion ability of a manipulator strongly depends on its Jacobian matrix. Therefore, general purpose functions were proposed to evaluate initial configurations based on data derived from some characteristics of the singular value decomposition of the Jacobian matrix. Additionally, a new task-specific function was designed which is based on some characteristics of a manipulability ellipsoid section.

Simulation results carried out on multi-dimensional planar and spacial pendula manipulators have shown that general-purpose functions evaluated at initial configurations weakly correlate with the lengths of the resulting loops in the configuration space. Therefore the functions are not suitable to distinguish initial configurations resulting in short loops. However, the new, dedicated functions display a strong and positive correlation especially when a given loop in a task-space is short and composed of a few privileged directions like in linear and rectangular paths.

\section{References}

Chiaverini, S., Oriolo, G. and Walker, I. (2008). Kinematically redundant manipulators, in B. Siciliano and O. Khatib (Eds.), Handbook of Robotics, Springer, Berlin, pp. 245-268.
Dixon, W.J. and Massey, F.J. (1969). Introduction to Statistical Analysis, McGraw-Hill, New York, NY.

Duleba, I. and Karcz-Duleba, I. (2016). A suboptimal solution of repeatable inverse kinematics in robot manipulators with a free entry configuration, Proceedings of the Mediterranean Conference on Control and Automation, Athens, Greece, pp. 563-568.

Duleba, I. and Karcz-Duleba, I. (2017). Suboptimal approximations in repeatable inverse kinematics for robot manipulators, Bulletin of the Polish Academy of Sciences: Technical Sciences 65(2): 209-218.

Duleba, I. and Karcz-Duleba, I. (2018). A comparison of methods solving repeatable inverse kinematics for robot manipulators, Archives of Computer Science, (to appear).

Duleba, I. and Opałka, M. (2013). On application of elastic band method to repeatable inverse kinematics in robot manipulators, Journal of Automation, Mobile Robotics and Intelligent Systems 7(4): 5-12.

Golub, G. and Reinsch, C. (1970). Singular value decomposition and least squares solutions, Numerische Mathematik 14(5): 403420.

Karpińska, J. (2012). Approximation of Algorithms for Robot Motion Planning, PhD thesis, Wrocław University of Technology, Wrocław, (in Polish).

Kendall, M. and Gibbons, J. (1990). Rank Correlation Methods, E. Arnold, London.

Klein, C., Chu-Jeng, C. and Ahmed, S. (1995). A new formulation of the extended Jacobian method and its use in mapping algorithmic singularities for kinematically redundant manipulators, IEEE Transactions on Robotics and Automation 11(1): 50-55.

Maciejewski, A. and Klein, C. (1989). The singular value decomposition: Computation and applications to robotics, International Journal of Robotics Research 8(6): 63-79.

Nakamura, Y. (1991). Advanced Robotics: Redundancy and Optimization, Addison Wesley, New York, NY.

Németh, B., Gáspár, P. and Péni, T. (2016). Nonlinear analysis of vehicle control actuations based on controlled invariant sets, International Journal of Applied Mathematics and Computer Science 26(1): 31-43, DOI: 10.1515/amcs-2016-0003.

Quinlan, S. and Khatib, O. (1993). Elastic bands: Connecting path and control, Proceedings of the IEEE International Conference on Robotics and Automation, Atlanta, GA, USA, Vol. 2, pp. 802-807.

Richter, S. and DeCarlo, R. (1983). Continuation methods: Theory and applications, IEEE Transactions on Automatic Control 28(6): 660-665.

Roberts, R. and Maciejewski, A. (1993). Repeatable generalized inverse control strategies for kinematically redundant manipulators, IEEE Transactions on Automatic Control 38(5): 689-699.

Spong, M. and Vidyasagar, M. (1989). Introduction to Robotics. Robot Dynamics and Control, MIT Press, Cambridge, MA. 
Tchoń, K., Karpińska, J. and Janiak, M. (2009). Approximation of Jacobian inverse kinematics algorithms, International Journal of Applied Mathematics and Computer Science 19(4): 519-531, DOI: 10.2478/v10006-009-0041-3.

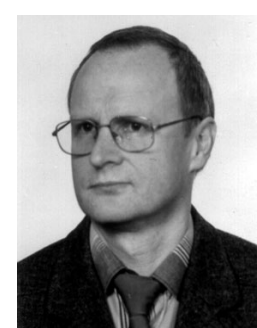

Ignacy Duleba received his $\mathrm{MSc}$ and $\mathrm{PhD}$ degrees in robotics from the Wrocław University of Technology (WUST) in 1986 and 1991, respectively. In 1999 he received his DSc degree from the Warsaw University of Technology. Currently, he is a full professor at the WUST. His scientific interests cover modelling and control of robots, as well as motion planning of nonholonomic systems.

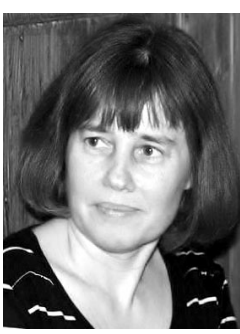

dynamical systems
Iwona Karcz-Duleba received her $\mathrm{MSc}, \mathrm{PhD}$ and DSc degrees from the Faculty of Electronics of the Wrocław University of Science and Technology (WUST) in 1988, 1992 and 2010, respectively. Currently, she is a professor at the WUST Faculty of Electronics, where she is the head of the Control, Modelling and Mechatronics Group. Her scientific activities include optimization, computational intelligence methods, modern heuristics, modelling and simulation of

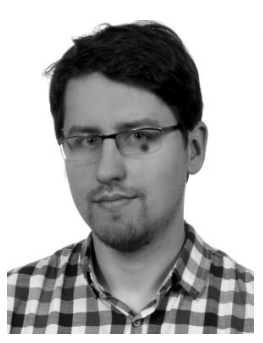

Arkadiusz Mielczarek is a $\mathrm{PhD}$ student in the Department of Cybernetics and Robotics of the Wrocław University of Science and Technology. He received his MSc degree in automatic control and robotics in 2016 from the same university. His research interests include nonholonomic motion planning, nonlinear control theory and signal processing (mainly in bio-medical applications).

Received: 21 August 2017

Revised: 20 November 2017

Accepted: 6 March 2018 